\title{
A New DOA Estimation Method Based on Spatial Structure of Array Signals
}

\author{
Mingyue Zhai \\ School of Computer and Information Engineering, Guangdong University Of Petrochemical \\ Technology, Maoming, Guangdong Province
}

Keyword: FDD; DOA; Signal space structure

\begin{abstract}
DOA estimation in FDD systems is a core issue for smart antennas. In this paper, an equidistant linear array is divided into two identical sub-arrays, and then a DOA matrix is constructed, the eigenvector matrix of the matrix is the array manifold of the signal. After eigenvalue decomposition of the manifold, the DOA of the signal can be estimated. In this paper, based on the theoretical analysis of computer simulation.
\end{abstract}

\section{Introduction}

With the development of social and economic life, mobile communication has become one of the main ways of communication. The rapid development of the market requires more newer technologies to ensure the capacity and quality of communication systems. In recent years, smart antennas are receiving more and more attention, which can effectively improve the system capacity and improve the system's signal-to-noise ratio. Smart antenna can improve the frequency utilization rate and further improve system's capacity when it is used in TDMA system. At the same time, smart antennas can also be combined with different duplexing modes. In the TDD mode, since the frequencies used by the uplink and downlink signals are the same, the directional emission of the downlink beam can use the parameters obtained by the uplink beamforming. In contrast, in the FDD method, the uplink signal and the downlink signal use different frequencies, and the downlink beamforming can not use the parameters obtained by the uplink beamforming. In this case, the DOA of the uplink signal is the only parameter that can be used and the only bridge between the uplink and downlink signals. Therefore, DOA estimation is a core issue of smart antenna technology in the FDD method ${ }^{[1]}$.

In document [2], two parallel linear antenna arrays are used to estimate the two-dimensional arrival angle of radar signals. In mobile communications, since the distance between the base station and the mobile station is much larger than the height of the base station antenna, the azimuth of the arrival signal is generally only considered irrespective of the elevation angle. Under the document[2], a linear array is divided into two sub-arrays of equal structure so as to construct a DOA matrix of the signal, and then the eigenvalue decomposition is used to obtain the signal arrival angle.

\section{Signal model}

Suppose we use a linear array, the number of elements is $M_{0}$. The antenna array is divided into two sub-arrays, wherein the array element number of the first sub-array is $(1,2, \ldots, M)$ and the array element number of the second sub-array is $(L, L+1, \ldots, L+M-1), M_{0}=M+L-1$. Among them, $L>=2$, is an integer.

Assuming that there are $K$ users $(K<M)$ at a certain time, the signal received by the $k$ th array element in the first sub-array can be expressed as:

$$
x_{k}(t)=\sum_{i=1}^{K} e^{j(k-1) w \frac{d}{c} \sin \theta_{i}} s_{i}(t)+n_{k}(t) \quad k=1,2, \ldots, M
$$


The signal received by the kth element of the second sub-array is expressed as:

$$
y_{k}(t)=\sum_{i=1}^{K} e^{j(k+L-2) w \frac{d}{c} \sin \theta_{i}} s_{i}(t)+n_{k+L-1}(t) \quad k=1,2, \ldots, M
$$

Where $s_{i}(t)$ is the modulation signal of the user $i, n_{k}$ is the noise received by the element $k$, $w$ is the carrier frequency.

Using matrix representation, $x_{k}(t)$ and $y_{k}(t)$ can be re-expressed as:

$$
\begin{aligned}
& X(t)=A S(t)+N_{x}(t) \\
& Y(t)=A \Phi S(t)+N_{y}(t)
\end{aligned}
$$

Where,

$$
\begin{aligned}
& X(t)=\left[x_{1}(t), x_{2}(t), \ldots, x_{M}(t)\right]^{T}, Y(t)=\left[y_{1}(t), y_{2}(t), \ldots, y_{M}(t)\right]^{T}, \\
& N_{x}(t)=\left[n_{1}(t), n_{2}(t), \ldots, n_{M}(t)\right]^{T}, N_{y}(t)=\left[n_{L}(t), n_{L+1}(t), \ldots, n_{L+M-1}(t)\right]^{T}, \\
& A=\left[a_{1}\left(\theta_{1}\right), a_{2}\left(\theta_{2}\right), \ldots, a_{K}\left(\theta_{K}\right)\right], \Phi=\operatorname{diag}\left[e^{j w \frac{d}{c}(L-1) \sin \left(\theta_{1}\right)}, e^{i w \frac{d}{c}(L-1) \sin \left(\theta_{2}\right)}, \ldots, e^{j w \frac{d}{c}(L-1) \sin \left(\theta_{K}\right)}\right] \\
& a_{i}\left(\theta_{i}\right)=\left[1, e^{j w \frac{d}{c} \sin \left(\theta_{i}\right)}, \ldots, e^{j w(M-1) \frac{d}{c} \sin \left(\theta_{i}\right)}\right]^{T}, \mathrm{i}=1,2, \cdots \cdots K .
\end{aligned}
$$

For this signal model, we make the following assumptions [4]: $M>K$, and linearly independent of $a_{1}\left(\theta_{1}\right), a_{2}\left(\theta_{2}\right), \ldots, a_{K}\left(\theta_{K}\right)$; noise is a Gaussian random variable with mean zero and variance $\sigma^{2}$ and statistical independence among the individual noises; That is,

$$
E\left\{N_{x}(t) N_{X}(t)^{H}\right\}=\sigma^{2} I, E\left\{N_{y}(t) N_{y}(t)^{H}\right\}=\sigma^{2} I .
$$

The matrix $P=E\left\{S(t) S(t)^{H}\right\}$ is positive definite.

From this signal model, we can see that we did not consider the multipath effect of users. Therefore, the mobile communication environment used in this model is an open suburban area or a rural area [5].

The autocorrelation matrix of signal $X(t)$ is expressed as $R_{X X}(t)=E\left\{X(t) X(t)^{H}\right\}$, and the cross-correlation matrix of signals $X(t)$ and $Y(t)$ is represented as $R_{Y X}(t)=E\left\{Y(t) X(t)^{H}\right\}$. Using equations (3) and (4), there are

$$
R_{X X}(t)=A P A^{H}+\sigma^{2} I, \quad R_{Y X}(t)=A \Phi P A^{H}+\Omega,
$$

where $P=E\left\{S(t) S(t)^{H}\right\}$. If $L>M$, all elements in $\Omega$ are zero. Otherwise, all but zero for elements $(1, L),(2, L+1),(M-L-1, M)$ are $\sigma^{2}$, we will change $\Omega$ to zero based on the estimated noise variance (see Algorithm Step 3), so in the following derivation we will omit $\Omega$.

After this assumption and treatment, we can use the method in literature [2] to get the following formula:

$$
R_{Y X} R_{X X 0}{ }^{*} A=A \Phi
$$

Where, $R_{X X 0}=A P A^{H}, R_{X X 0}{ }^{*}$ represent the pseudo-inverse matrix [6], which can be expressed as $R_{X X 0}{ }^{*}=\sum_{i=1}^{K} \frac{1}{\mu_{i}-\sigma^{2}} V_{i} V_{i}^{H}, \mu_{i}$ (sorted in descending order of absolute value) and $V_{i}$ are the 
eigenvalues and eigenvectors of $R_{X X}$, respectively. Since the matrix $\Phi$ is a diagonal matrix, equation (5) shows that the column vector of array manifold $\mathrm{A}$ is the eigenvector of matrix $R_{Y X} R_{X X 0}{ }^{*}$, and the corresponding diagonal elements of diagonal matrix $\Phi$ are the eigenvalues of matrix $R_{Y X} R_{X X 0}{ }^{*}$.

Let $R=R_{Y X} R_{X X 0}{ }^{*}$, and call it the DOA matrix [2] (from the above equation, $R$ has only $K$ non-zero eigenvalues ${ }^{[1]}$ ).

\section{DOA Algorithm Based on Signal Spatial Features}

According to eigenvalue decomposition of DOA matrix, we can get the following DOA estimation algorithm:

According to the received signal, estimating the auto-correlation matrix $R_{X X}$ of $X(t)$ and cross-correlation matrix $R_{Y X}$ of $X(t), Y(t)$. In this paper, we use the progressive unbiased estimator $^{[7]}$ :

$$
R_{X X}=\frac{1}{N} \sum_{t=1}^{K} X(t) X(t)^{H}, R_{Y X}=\frac{1}{N} \sum_{t=1}^{K} Y(t) X(t)^{H} .
$$

Calculating the eigenvalue $\left(\mu_{1}, \mu_{2}, \ldots, \mu_{N}\right)$ and eigenvector $\left(\left(V_{1}, V_{2}, \ldots, V_{N}\right)\right)$ of $R_{X X}$;

From formula(18), we can see that the user's number $K$ is the larger number in $\mu_{1}, \mu_{2}, \ldots, \mu_{N}$ (the smaller eigenvalue corresponds to the variance of the noise, so the variance of noise can be taken as the average of the smaller eigenvalues so that the $\Omega$ in the formula(8)can be changed into zero matrix);

Constructing DOA matrix $R$ according to the formula (19);

Calculating the eigenvalues and eigenvectors of $R$, from which selecting $K$ larger eigenvalues $\varepsilon_{i}$ and their corresponding eigenvectors $Z_{i}$;

Constructing search function

$$
f(\theta)=\sum_{i=1}^{K} \frac{1}{\left|a(\theta)-\frac{Z_{i}}{Z_{i}(1)}\right|^{2}}
$$

Where, $Z_{i}(1)$ is the first element of the eigenvector $Z_{i}$. The angle $\theta$ corresponding to the peak value of $f(\theta)$ is the arrival angle of the user $i$.

\section{Numerical Simulation}

For this algorithm, we conducted a numerical simulation to verify its validity.

Test one: this test is used to verify the algorithm and estimate the performance of multi-user signal DOA. Array element is 9, user's number is 4, received signal is BPSK signal, carrier frequency is $f_{c}=900 \mathrm{MHz}$, signal's baud rate is $2 f_{c}$, sampling points is 2000 , signal-noise ratio is $20 \mathrm{~dB}$. The angle of incidence for the 4 users is $(-60,-50,20,40)$. The results is shown as the Fig.1, the vertical axis of the figure is in units of $\mathrm{dB}$, the peak corresponding to the abscissa is the user's DOA. A total of 10 trials were conducted.

Test two:this test is used to verify the resolution ratio. User's number is 2, incidence angle is( -2 , 2). The remaining parameters are consistent with the test one, and the results is shown in Fig. 2.

\section{Conclusion}

In this paper, a straight line is divided into two identical sub-arrays, and then DOA is estimated 
according to the spatial characteristics of the array signal. In this method, the eigenvalue decomposition of the signal autocorrelation matrix and the cross-correlation matrix is fully used to construct the DOA matrix of the array signal. The eigenvectors corresponding to the non-zero eigenvalues of the matrix are the array manifolds, The user's angle of arrival can be found by finding the extremum of the search function. Computer simulation results show that this method is feasible and the resolution is also high.

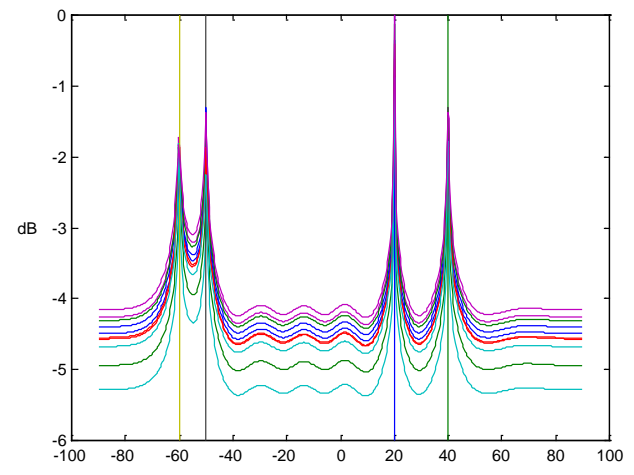

Figure 1. 4 users

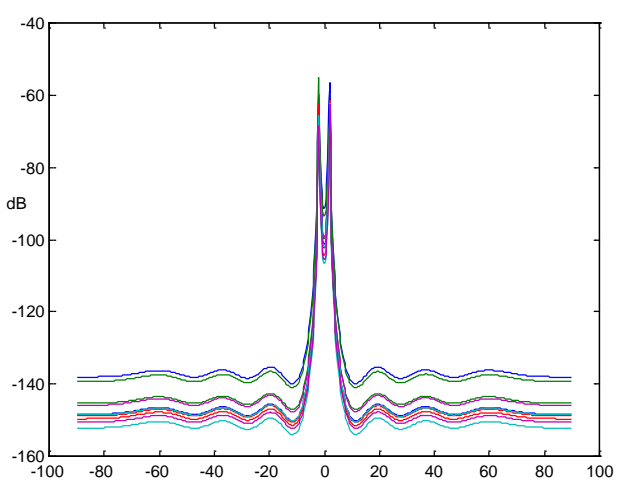

Figure 2. 2 users

\section{References}

[1] M.L. Yao,L. Jin and Q.Y. Yin.Estimation of Direction of Arrivals in Cumulative Volume Based on Spatial Features in Multiuser Cycles[J].Journal of Communication,2015,5,(21):36-40.

[2] Q. Y. Yin, R.W. Newcomb, L. H. Zou. Estimating 2-D Angles of Arrival via Two Parallel Linear Arrays[J]. Proc. Of IEEE ICASSP, 2009, (4):2803 2806.

[3] LAL C. Godara. Applications of Antenna Arrays to Mobile Communications, Part I: Performance Improvements, Feasibility, and System Considerations[J], Proceedings of The IEEE, July 20077, Vol. 85, No. 7:1031-1060.

[4] X.D.Zhang.Modern signal processing[M].Beijing:Tsinghua University Press, 1995,5.

[5] Y.A. Liu,etc.Broadband wireless access with wireless LAN[M].Beijing:Beijing University of Posts and Telecommunications Press,2016,12.

[6] X.H. Wu,C.D. Chen and Z.F. Qian.Matrix theory[M].Shanghai:Tongji University Press, 1994,3.

[7] M. Wax, T. Shan, T. Kailath. Spatiao-Temporal Spectral Analysis by Eigenstructrue Method[J]. IEEE Trans. On ASSP, Aug. 2014, vol. ASSP-31:817-827. 